\section{Images in \\ Cardiovascular Medicine}

\section{Check for updates}

\section{OPEN ACCESS}

\section{Received: Apr 19, 2019}

Revised: Jun 7, 2019

Accepted: Jul 4, 2019

\section{Correspondence to}

Soo Wan Kim, MD, PhD

Department of Internal Medicine, Chonnam National University Medical School, 42, Jebong-ro, Dong-gu, Gwangju 61469, Korea.

E-mail: skimw@chonnam.ac.kr

Copyright $(2019$. The Korean Society of Cardiology

This is an Open Access article distributed under the terms of the Creative Commons Attribution Non-Commercial License (https:// creativecommons.org/licenses/by-nc/4.0) which permits unrestricted noncommercial use, distribution, and reproduction in any medium, provided the original work is properly cited.

\section{ORCID iDs}

Chang Seong Kim (ID)

https://orcid.org/0000-0001-8753-7641

Hyung Yoon Kim (D)

https://orcid.org/0000-0002-0692-5640 Kyo Seon Lee (ID)

https://orcid.org/0000-0001-7397-4680 Eun Hui Bae (D)

http://orcid.org/0000-0003-1727-2822

Seong Kwon Ma (D)

https://orcid.org/0000-0002-5758-8189 Soo Wan Kim (D)

https://orcid.org/0000-0002-3540-9004

\section{Funding}

This work was supported by the National Research Foundation of Korea (NRF) grant funded by the Korea government (Ministry of Science and ICT) (NRF-2017M3A9E8023018)

\title{
Vascular Stent Migration to Right Ventricle
}

\author{
Chang Seong Kim (1), MD', Hyung Yoon Kim (i), MD², Kyo Seon Lee $\mathbb{1}, M^{3}$,

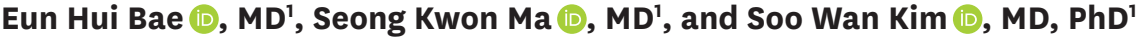 \\ 'Department of Internal Medicine, Chonnam National University Medical School, Gwangju, Korea \\ ${ }^{2}$ Department of Cardiovascular Medicine, Chonnam National University Hospital, Gwangju, Korea \\ ${ }^{3}$ Department of Thoracic and Cardiovascular Surgery, Chonnam National University Medical School, \\ Gwangju, Korea
}

A 62-year-old woman with diabetes mellitus, hypertrophic cardiomyopathy, and end-stage renal disease was undergoing chronic hemodialysis via a right radio-cephalic arteriovenous graft. She required hospitalization due to fever, dyspnea, and right shoulder pain. One year earlier, she had undergone balloon angioplasty, followed by deployment of an axillary vein stent $(6 \times 80 \mathrm{~mm})$ due to central stenosis. Upon admission, computed tomography revealed right shoulder and psoas muscle abscesses and a pulmonary thromboembolism. Coincidently, during the work-up, a metallic foreign body was detected in the right ventricle (RV), suggestive of a migrated vascular stent (Figure 1A-C). Transthoracic echocardiography revealed a migrated vascular stent in the RV cavity, impacted between the tricuspid valve (TV) septal leaflet and the RV apex (Figure 1D). After antibiotic treatment of the abscesses, the stent was successfully removed, without TV or RV injury, through the right atrium (Figure 2A and B).
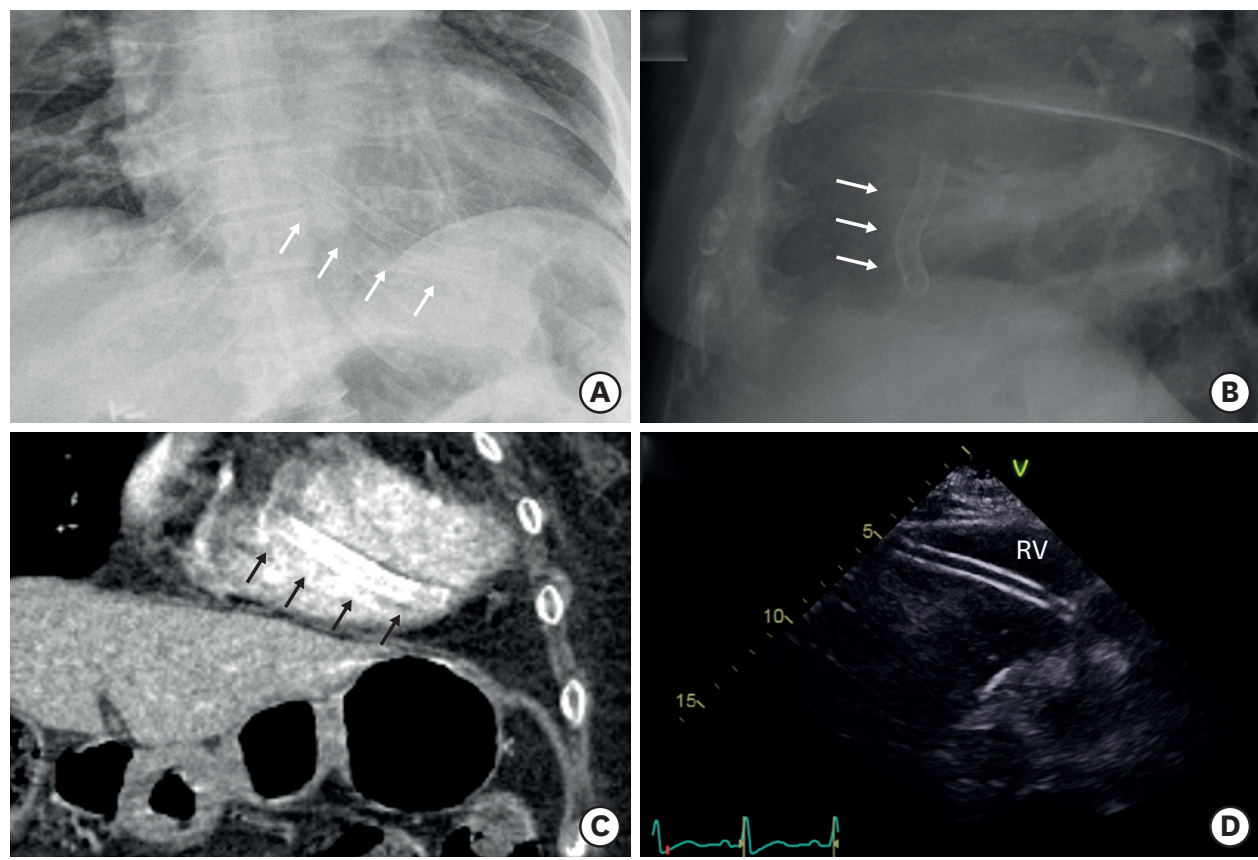

Figure 1. (A, B) Anterior and lateral view of chest $\mathrm{X}$-ray showing the stent presents within the anterior inferior chest (arrows). (C) Coronal view of computed tomography showing the migrated stent in the RV (arrows). (D) Transthoracic echocardiography showed a migrated vascular stent in the RV. $\mathrm{RV}=$ right ventricle. 
and by the Basic Science Research Program through the NRF funded by the Ministry of Education (NRF-2018R1D1A1B07042999).

\section{Conflict of Interest}

The authors have no financial conflicts of interest.

\section{Author Contributions}

Conceptualization: Kim CS, Kim SW; Methodology: Kim CS; Supervision: Ma SK, Kim SW; Validation: Kim HY, Lee KS; Writing - original draft: Kim CS; Writing - review \& editing: Kim HY, Lee KS, Bae EH, Ma SK, Kim SW.
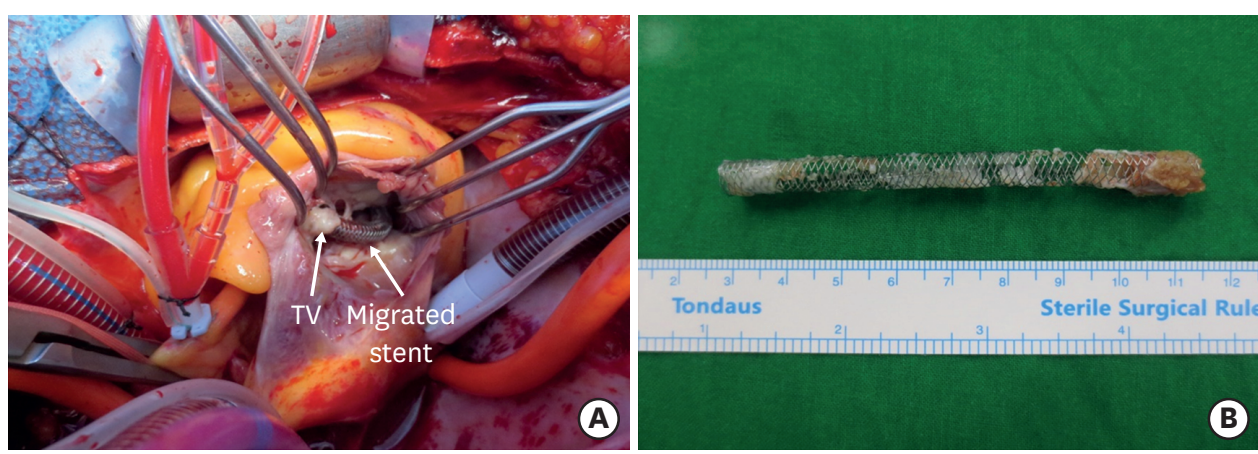

Figure 2. (A) The right atrium was opened, and the stent was attached with TV in the right ventricle. (B) An extracted stent.

$\mathrm{TV}=$ tricuspid valve

Despite postoperative complications of pancreatitis and ischemic colitis, she was discharged 2 weeks after the operation. A migrated venous stent could be moved unimpeded up to the right atrium, ventricle, and pulmonary arteries because of the direction of venous blood flow as well as the gradual increase in the vein diameter to the heart. ${ }^{1)}$ However, stent-related factors (too small stent or inadequate ballooning), variations in the diameters of central vein with respiration and cardiac impulse, or excess shoulder movement resulting in stent detachment from the axillary vein might have been the cause of stent migration in this case. A retrospective image review suggested that the stent may have migrated within 2 months after its insertion; stent migration was identified late because serious acute complications, such as cardiogenic shock, RV rupture, TV injury, and arrhythmia, did not occur. Although stent migration is a rare complication of stent placement that can occur at the time of placement or later, a vascular stent inserted into a central vein stenotic lesion should be periodically monitored for migration. ${ }^{2)}$

\section{REFERENCES}

1. Sequeira A. Stent migration and bail-out strategies. J Vasc Access 2016;17:380-5. PUBMED | CROSSREF

2. Ho JM, Kahan J, Supariwala A, et al. Vascular stent fracture and migration to pulmonary artery during arteriovenous shunt thrombectomy. J Vasc Access 2013;14:175-9.

PUBMED | CROSSREF 